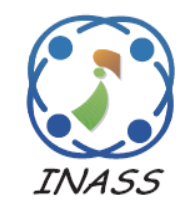

\title{
TLBO-Based Routing Approach for Wireless Sensor Networks
}

\author{
Asmae El Ghazi ${ }^{1 *} \quad$ Belaîd Ahiod $^{1} \quad$ Mohammed Abbad $^{1}$ \\ ${ }^{1}$ LRIT, Associated Unit to CNRST (URAC 29) Faculty of Sciences, \\ Mohammed $V$ University in Rabat, Morocco \\ * Corresponding author's Email: as.elghazi@gmail.com
}

\begin{abstract}
Routing in Wireless Sensor Networks (WSN) differs from routing in classical networks, as in WSN links are unreliable. Indeed, there is no infrastructure and the sensors have limited energy resources and low processing capacity. Routing problems in many cases are considered NP-hard problems. In these cases, metaheuristics can be the best solution for this kind of problems. Thus, many metaheuristics are used for routing in WSNs. Ant colony optimization (ACO) is among the most used metaheuristics, as it showed an excellent performance for routing. Due to the adaptation difficulties related to the ACO parameters, in this paper we propose a new optimization approach for routing problem using the Teaching Learning Based Optimization (TLBO). TLBO is a recent nature inspired metaheuristic based on the influence of the teacher in the class and learners interactions. In this work, TLBO algorithm is used for routing as it was proposed for continuous optimization problems, despite the fact that the routing in WSNs is a discrete optimization problem. Our proposed approach is shown to be competitive with ACObased approaches in terms of energy consumption and WSN lifetime. The experiments performed in varied scenarios and the results found, illustrate the efficiency of the TLBO approach against the well-known ACO approaches: Energy Efficient Ant Based Routing (EEABR) Algorithm and the Improved Ant Colony Optimization Routing Protocol (IACO).
\end{abstract}

Keywords: Wireless sensor network, Metaheuristic, Routing, Ant colony optimization, Teaching-learning-based optimization.

\section{Introduction}

Wireless Sensor Networks represent an attractive research area due to several factors such as the resource-constrained nature of sensor nodes, interference, energy consumption and a large number of both commercial and military applications that this technology offers [1]. Successful network design and deployment include understanding and modelling several problems related to these factors, which determine the accessible extent and data rate of a WSN, as well as cost and battery lifetime [2]. Therefore, studies, intended to researchers and graduate fields related to operations research, applied mathematics and computer science, give some highlights on a number of representative network problems in WSN and focus on their respective optimization problems [3].
NP-hard optimization problems induce the necessity of optimization methods like metaheuristics. In WSN, the most studied optimization problem is the routing problem, as it is a major challenge.

To resolve routing in wireless sensor networks a diverse range of metaheuristic algorithms are used including Artificial Bee Colony (ABC) [4], Genetic Algorithms (GA) [5], Particle Swarm Optimization (PSO) [6], Harmony Search (HS) [7] and Ant Colony Optimization (ACO) [8], etc.

The ACO metaheuristic has been successfully applied to solve routing problems in WSN $[9,10]$. Some examples of ant based application include: Sensor-driven Cost-aware Ant Routing (SC), the Flooded Forward Ant Routing (FF) algorithm [11], the Flooded Piggy-backed Ant Routing (FP) algorithm, the Adaptive ant-based Dynamic Routing (ADR) [12], the Adaptive Routing (AR) Improved Adaptive Routing (IAR) algorithm [13], E\&D 
ANtraTS [14], Energy Efficient Ant Based Routing (EEABR) Algorithm [15] and the Improved Ant Colony Optimization Routing Protocol (IACO) [8].

A new efficient metaheuristic has recently been developed: Teaching Learning Based Optimization (TLBO) [16, 17]. TLBO algorithm is based on teacher influence on learners' output in a class. This metaheuristic is characterized by the reduced number of parameters, as the complexity of the known metaheuristics is the number of parameters used. In this paper, we propose a new optimization approach for the routing problems using TLBO as it was proposed for a continuous optimization problems. To demonstrate the efficiency of the proposed TLBO protocol, many simulations have been performed in MATLAB, using various ranges of nodes, a different detection zone area, a random source nodes and a random deployment. The obtained results demonstrate that our based model gives a good performance in terms of energy consumption, data delivery and reliability.

The remaining of the paper is organized as follows: Section 2 presents the routing approach based on the ACO. Section 3 describes the main steps of our routing approach based on the TLBO and an illustrative example is given in the same section. Section 4 shows the performance evaluation of our algorithm. Finally, Section 5 concludes our work.

\section{Routing approach based on the ACO}

The authors in [8] have proposed a routing approach based on ant colony optimization (IACO), which has provided good results compared with others ant colony optimization approaches. The ant approach proposed is based on the following algorithm of Ant colony optimization, used to find a good path from the source node to the sink.

\subsection{Ant colony optimization algorithm}

The ant colony has a high ability to explore and exploit their environment and using it as a medium to share information. M. Dorigo and G. Di Caro have been inspired by ant colonies in nature in order to create ant colony optimization algorithms [18]. The ACO algorithm basic steps are presented in Algorithm 1.

An ant moves from a node $s$ to a node $r$ with the highest probability value. Every ant walking between nodes lets an amount of pheromone $\delta \tau_{i j}$. This quantity of pheromone changes following environmental factors (wind, sun...), which are presented by the evaporation rate parameter $\rho$.

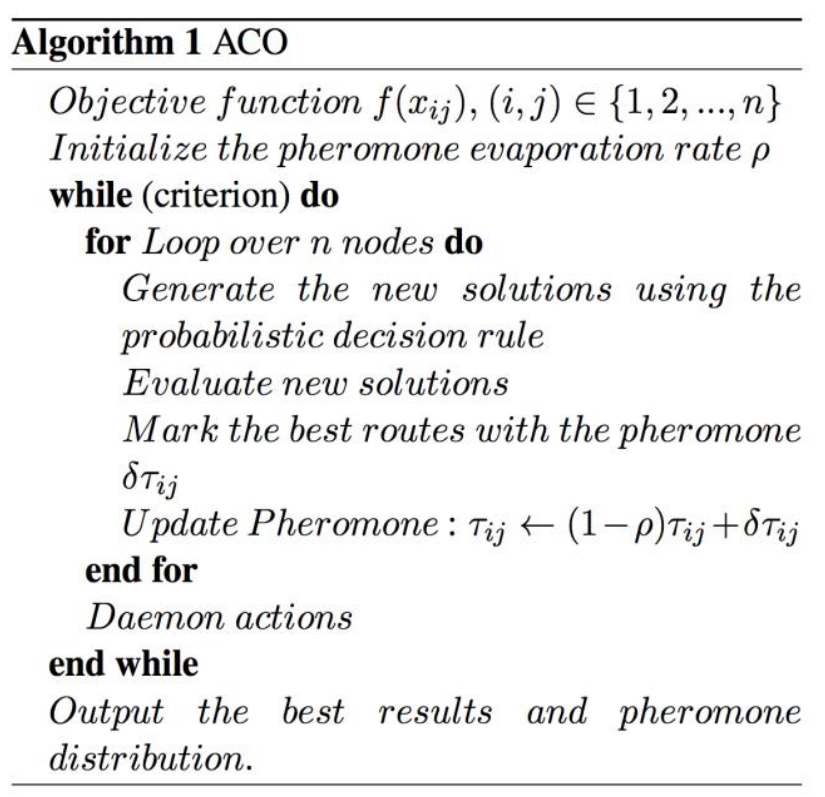

\subsection{Routing using ant colony optimization}

\subsubsection{Improved ant colony optimization}

The ACO approach is proposed for routing in flat networks with static sensors and sink, in order to minimize the energy consumption. When an event is detected the information is transmitted to the sink separated on $N$ parts. The ants use the probabilistic decision rules Eq. (1) in the path from source to the base station.

$$
\begin{aligned}
& P_{k}(r, s)= \\
& \left\{\begin{array}{cc}
\frac{[\tau(r, s)]^{\alpha} \cdot[\eta(r, s)]^{\beta} \cdot[\delta(r, s)]^{\gamma}}{\sum_{r \in R_{S}[\tau(r, s)]^{\alpha} \cdot[\eta(r, s)]^{\beta} \cdot[\delta(r, s)]^{\gamma}}} & \text { if } k \notin t a b u^{r} \\
0 & \text { otherwise }
\end{array}\right.
\end{aligned}
$$

Where $\tau(r, s)$ is the pheromone value between node $s$ and $r, \eta$ is the first heuristic value related to the energy of nodes. $R_{S}$ are the receiver nodes, the second heuristic value is $\delta$ used to distinguish the best neighbor. $\alpha, \beta$ and $\gamma$ are the control parameters relative to the pheromone influence and the heuristics information. $t a b u^{r}$ is the list of packet identities already received by node $r$. This approach gives good results, compared to Okdem et al. approach [9]. However, the novel optimization technique based on TLBO proposed in this paper promises better results as demonstrated in the following sections.

\subsubsection{Energy-efficient ant-based routing}

Camilo et al. proposed an Energy Efficient Ant Based Routing (EEABR) Algorithm, which is based $\mathrm{ACO}$, to improve the energy efficiency of WSNs 
and maximize the network lifetime [15]. This routing algorithm, found the paths between the source nodes and the sink by forward ants, which select next hop according to the residual energy of neighbors and the amount of pheromone left by backward ant during its journey. Meanwhile, the number of visited nodes, is introduced in this phase, which makes those nodes closer to sink node have more pheromone trail, so that forward ants could reach the sink node more efficiently. However, during the early period of transmitting packets, the forward ants cannot find the optimal or near optimal paths due to the little difference of the amount of pheromone trail stored in routing table.

The algorithm uses a good strategy considering energy levels of the nodes and the lengths of the routed paths, which have a great contribution to balancing the energy consumption of nodes and reducing the time delay. Considering this fact, we have compared the performance results of our TLBO approach to the results of the EEABR algorithm.

\section{Our routing approach based on the TLBO}

TLBO approach is a new optimization technique for routing problem in WSN, proposed to overcome ant- approach drawbacks such as the parameters tuning difficulties and the processing time, considering the sensor limitations. The routing process begins by the initialization of the population of paths, then finding the optimum path using TLBO algorithm described in the next section, then sends data parts through it.

\subsection{The TLBO algorithm}

Inspired by the teacher's influence in the class and learners interaction, Rao et al. have developed in 2012 the Teaching learning based optimization algorithm [17]. This method has outperformed some of the commonly used metaheuristics regarding continuous nonlinear numerical optimization problems. It could be split into two basic parts: Teacher phase and Learner phase. TLBO has been used successfully for many multi-objective problems [19, 20]. Algorithm 2 describes TLBO process.

TLBO algorithm starts by the initialization of the population and the termination criterion, and then researches the best solution using the teacher and the learner phases. At the end, it verifies if the termination criterion is satisfied or not.

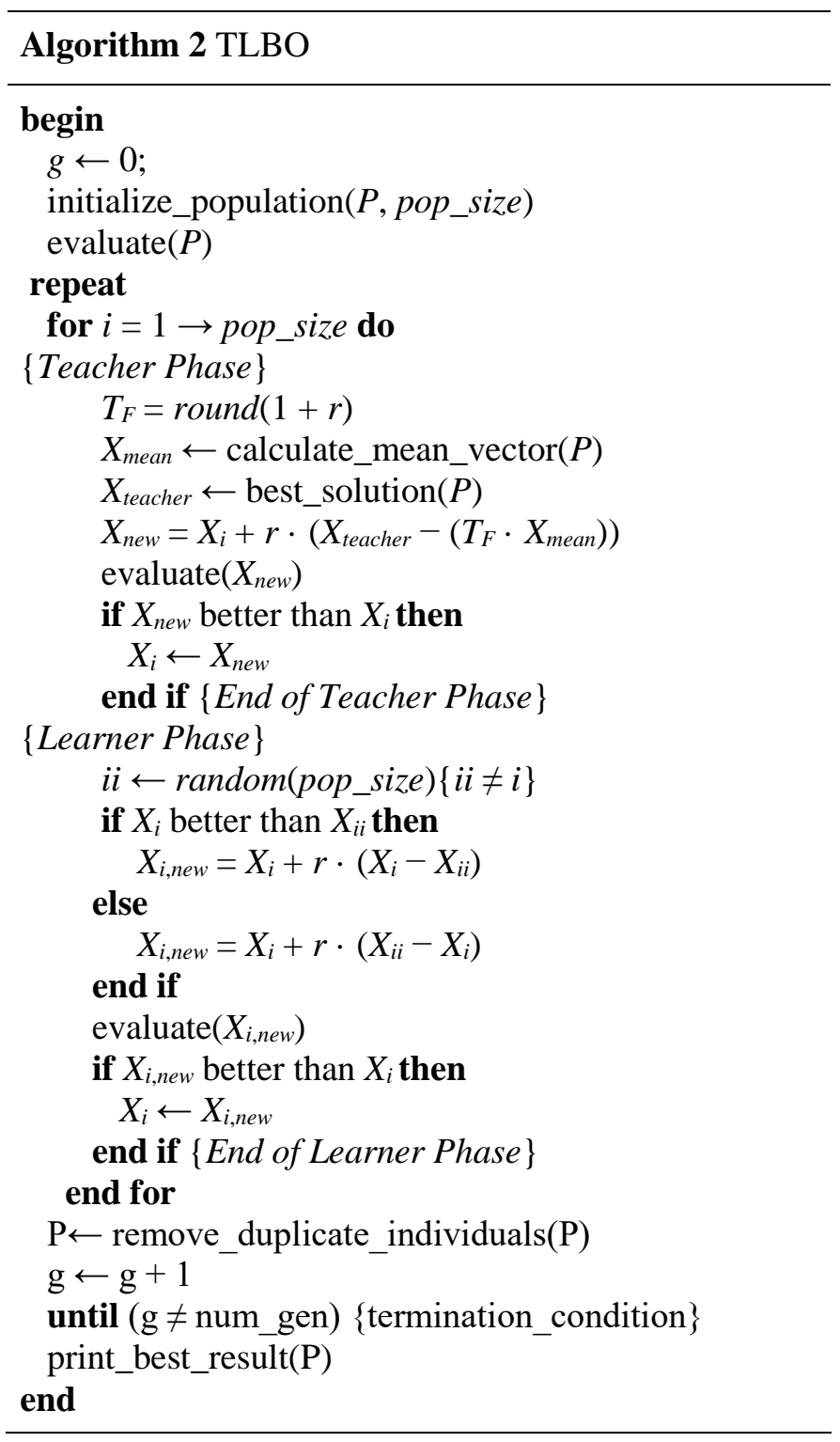

\subsection{Adapting TLBO for routing in WSN}

We implement TLBO for routing problem following five steps. In the first step, we initialize the optimization parameters; in the second one we generate the initial population. The third step represents the teacher phase and the fourth one presents the learner phase. Finally, in the last step after verification, we stop the process or we restart.

Step 1: Initialization of the optimization parameters considered for routing problems and definition of the objective function.

The node detecting an event collects information about available routes that lead to the base station. By requesting neighbors for a definite time, a number of paths form the initial population:

Paths $=\left\{p_{1}, \ldots, p_{i}, \ldots, p_{m}\right\}$, where every path $p_{i}$ is formed a $p_{i}=\left\{n_{s}, \ldots, n_{k}, \ldots, n_{\operatorname{sink}}\right\}$. 
Population size $(P n)$ : depends on the waiting time that the source node fixed before requesting neighbors.

Number of generations $(G n)$ : varied depending on population size (if the population size is small the algorithm converges faster).

$$
\text { Number of design variables }(D n)=3
$$

Optimization problem: Minimize

$$
f\left(p_{i}\right)=\frac{\sum_{1}^{n} E\left(p_{i}\right) \cdot \sum_{1}^{n} d\left(p_{i}\right)}{n}
$$

Where $p_{i}$ is the corresponding $i^{\text {th }}$ element in $P n, n$ is length of $p_{i}, d\left(p_{i}\right)$ is approximate delay for $p i$, and $E\left(p_{i}\right)$ is approximate energy consumed by $p_{i}$.

Step 2: The energy consumption, number of hops and delay represent design variables. According to those ones a random population is generated, where the number of paths corresponds to the number of learners. This population is expressed as:

$$
\text { population }=\left(\begin{array}{ccc}
x_{1,1} & x_{1,2} & x_{1,3} \\
\vdots & \ddots & \vdots \\
x_{m, 1} & x_{m, 2} & x_{m, 3}
\end{array}\right)
$$

The respective objective values are:

$$
\left(\begin{array}{c}
f_{1} \\
\vdots \\
f_{i} \\
\vdots \\
f_{m}
\end{array}\right)
$$

Step 3 (Teacher phase): The mean of each population column:

$$
\text { Mean }=\left(\begin{array}{lll}
m_{1} & m_{2} & m_{3}
\end{array}\right)
$$

Teacher is the best solution for this iteration:

$$
\begin{gathered}
X_{\text {teacher }}=\min _{1 \leq i \leq m}\left(f\left(X_{i}\right)\right), \\
X_{i}=\left[x_{i, 1}, x_{i, 2}, x_{i, 3}\right]
\end{gathered}
$$

The difference between both values is expressed as:

$$
\text { Difference }_{D}=r\left(X_{\text {teacher }}-\text { TFMean }\right)
$$

According to this difference a new solution is calculated $X_{\text {new }} . X_{\text {new }}$ is accepted if its objective value is better than $X_{\text {old }}$.

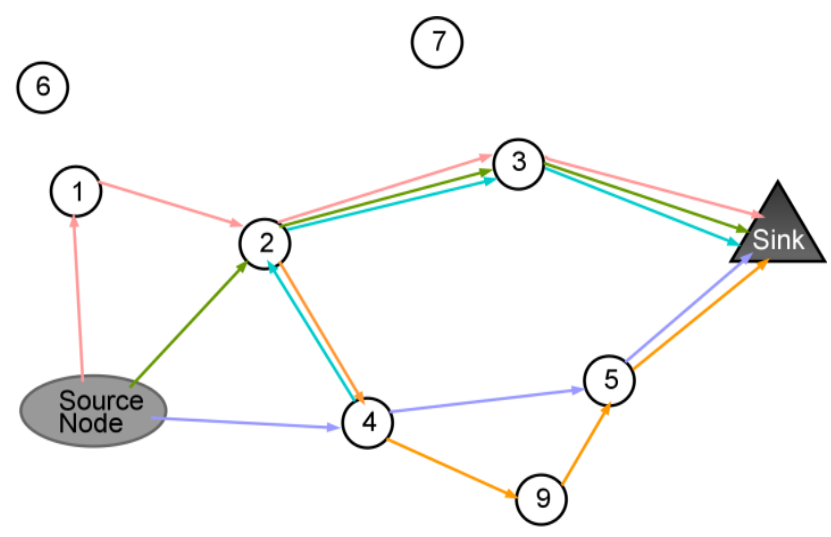

Figure.1 Initialization of population

Step 4 (Learner phase) Select a random $X_{j}$ from population, compare it to the current one $X_{i}$, then a new solution is determined by using Eq. (3).

$\left\{X_{\text {new }, i}=X_{\text {old }, i}+r_{i}\left(X_{i}-X_{j}\right)\right.$, if $f\left(X_{i}\right)<f\left(X_{j}\right)$ $\left\{X_{\text {new }, i}=X_{\text {old }, i}+r_{i}\left(X_{j}-X_{i}\right)\right.$, if $f\left(X_{j}\right)<f\left(X_{i}\right)$

Step 5: If the number of generations is achieved stop process, otherwise go to step 3 .

\subsection{Illustrative example}

In this example, 10 nodes are deployed randomly. The source node s sends request packets to neighbors and then initializes the population (see Fig. 1).

Nodes 1,2 and 4 receive a request from the source node. Each one checks the routing table, and then requests their neighbors and so on until reaching the sink. After this procedure, a population of 5 paths is generated. (See Fig. 1)

\section{- Population:}

Initial population composed by:

$$
\begin{aligned}
& p_{1}=\{S, 1,2,3, \text { Sink }\} \\
& p_{2}=\{S, 2,3, \text { Sink }\} \\
& p_{3}=\{S, 4,5, \text { Sink }\} \\
& p_{4}=\{S, 2,4,9,5, \text { Sink }\} \\
& p_{5}=\{S, 4,2,3, \text { Sink }\}
\end{aligned}
$$

\section{- Design Variables:}

The considered design variables are:

- Consumed Energy: $E\left(p_{i}\right), 0<i<6$

- Delay: $d\left(p_{i}\right)$

- Number of hops in $p_{i}$

For $p_{1}$ :

$E\left(p_{1}\right)=0.0058$ 
$d\left(p_{1}\right)=2.9645$

Number of hops in $p_{1}$ is 5

After the same calculation for all paths $p_{i}$, the population is:

$$
\text { Pop }=\left(\begin{array}{ccc}
0.0058 & 2.9645 & 5 \\
0.0046 & 2.3716 & 4 \\
0.0047 & 2.3716 & 4 \\
0.0071 & 3.5574 & 6 \\
0.0060 & 2.9645 & 5
\end{array}\right)
$$

\section{- TLBO implementation:}

$\checkmark$ Objective function:

$$
f=\left(\begin{array}{l}
0.0034 \\
0.0027 \\
0.0028 \\
0.0042 \\
0.0036
\end{array}\right)
$$

$\checkmark$ Iteration 1:

\section{\{Teacher Phase\}}

$$
\begin{aligned}
& \operatorname{Min}(f)=0.0027 \\
& \Rightarrow \quad p_{2}=\{S, 2,3, \text { Sink }\} \\
& T_{F}=1 \text { and } r=0.1968 \\
& f\left(X_{\text {new }, 1}\right)=0.0027 \\
& f\left(X_{\text {old }, 1}\right)=0.0034 \\
& f\left(X_{\text {new }, 1}\right)<f\left(X_{\text {old }, 1}\right) \\
& \Rightarrow \quad X_{1}=X_{\text {new }, 1}
\end{aligned}
$$$$
\operatorname{Pop}(2)=\left[\begin{array}{lll}
0.0046 & 2.3716 & 4
\end{array}\right]
$$$$
X_{\text {Teacher }}=\left[\begin{array}{lll}
0.0046 & 2.3716 & 4
\end{array}\right]
$$$$
\text { Mean }=\left(\begin{array}{lll}
0.0056 & 2.8459 & 5.000
\end{array}\right)
$$$$
\text { Difference }_{D}=[-0.0007-0.3216-0.6781]
$$$$
X_{\text {new }, 1}=\left[\begin{array}{lll}
0.0051 & 2.6429 & 5.0000
\end{array}\right]
$$

\section{\{Learner Phase $\}$}

Selecting a random solution $X_{j}(i \neq j)$

$j=2$

$X_{1}=\left[\begin{array}{lll}0.0051 & 2.6429 & 5.0000\end{array}\right]$

$X_{2}=\left[\begin{array}{lll}0.0046 & 2.3716 & 4.0000\end{array}\right]$

$f\left(X_{1}\right)=0.0027$

$f\left(X_{2}\right)=0.0027$

$r=0.0246$

$X_{n e w, 1}=X_{1}-r\left(X_{2}-X_{1}\right)$

$\Rightarrow \quad X_{\text {new }, 1}=\left[\begin{array}{lll}0.0053 & 2.7404 & 5.0000\end{array}\right]$

$\operatorname{Pop}(1)=X_{\text {new }, 1}$

$$
\text { Pop }=\left(\begin{array}{lll}
0.0053 & 2.7404 & 5.0000 \\
0.0046 & 2.3716 & 4.0000 \\
0.0047 & 2.3716 & 4.0000 \\
0.0071 & 3.5574 & 6.0000 \\
0.0060 & 2.9645 & 5.0000
\end{array}\right)
$$

Iteration 2:

\section{\{Teacher Phase\}}

$$
\begin{aligned}
& T_{F}=2 \text { and } r=0.6799 \\
& \text { Difference }_{D}=[-0.0044-2.1965 \\
& -4.0794] \\
& \Rightarrow \quad X_{\text {new }, 2}=\left[\begin{array}{lll}
0.0002 & 0.1751 & 4.0000
\end{array}\right] \\
& f\left(X_{\text {new }, 2}\right)=8.8305 e^{-06} \\
& f\left(X_{\text {old }, 2}\right)=0.0027 \\
& f\left(X_{\text {new }, 2}\right)<f\left(X_{\text {old }, 2}\right) \\
& \Rightarrow X_{2}=X_{\text {new }, 2}
\end{aligned}
$$

\section{\{Learner Phase\}}

Selecting a random solution $X_{j}(i \neq j)$

$j=3$

$X_{2}=\left[\begin{array}{lll}0.0002 & 0.1751 & 4.0000\end{array}\right]$

$X_{3}=\left[\begin{array}{lll}0.0047 & 2.3716 & 4.0000\end{array}\right]$

$f\left(X_{2}\right)=8.8305 e^{-06}$

$f\left(X_{3}\right)=0.028$

$f\left(X_{3}\right)>f\left(X_{2}\right)$

$r=0.4299$

$X_{\text {new }, 2}=X_{2}-r\left(X_{3}-X_{2}\right)$

$\Rightarrow \quad X_{\text {new }, 2}=\left[\begin{array}{lll}-0.0017 & -0.7691 & 4.0000\end{array}\right]$

$\operatorname{Pop}(2)=X_{\text {new }, 2}$

$$
\text { Pop }=\left(\begin{array}{ccc}
0.0053 & 2.7404 & 5.0000 \\
-0.0017 & -0.7691 & 4.0000 \\
0.0047 & 2.3716 & 4.0000 \\
0.0071 & 3.5574 & 6.0000 \\
0.0060 & 2.9645 & 5.0000
\end{array}\right)
$$

After a number of generations:

$$
\begin{gathered}
\text { Pop }=\left(\begin{array}{ccc}
0.0006 & 0.3834 & 5.0000 \\
0.0007 & 0.7529 & 4.0000 \\
-0.0004 & -0.0997 & 4.0000 \\
-0.0007 & -0.3933 & 6.0000 \\
0.0013 & 0.8347 & 5.0000
\end{array}\right) \\
f=1.0 e^{-03} *\left(\begin{array}{l}
0.0492 \\
0.1247 \\
0.0100 \\
0.0491 \\
0.2204
\end{array}\right) \\
\Rightarrow \operatorname{Min}(f)=0.0100 * 1.0 e^{-03}
\end{gathered}
$$

Best solution is:

$$
\begin{aligned}
& \operatorname{Pop}(3)=\left(\begin{array}{lll}
0.0065 & 3.2869 & 5.5438
\end{array}\right) \\
& \Rightarrow p_{3}=\{S, 4,5, \text { Sink }\}
\end{aligned}
$$

\section{- Data transmission:}

Source node splits data to $N$ parts and sent packets to the sink through the chosen path $p_{3}$ as described in Fig. 2.

If a node of $p_{3}$ fails, the previous node in the path must search a new path, using the same process as source node.

$$
\text { Mean }=\left(\begin{array}{lll}
0.0055 & 2.8011 & 5.0000
\end{array}\right)
$$


(6)

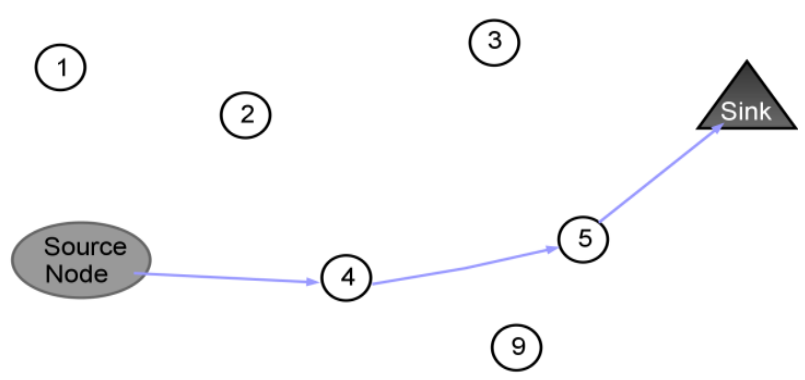

Figure.2 Data transmission
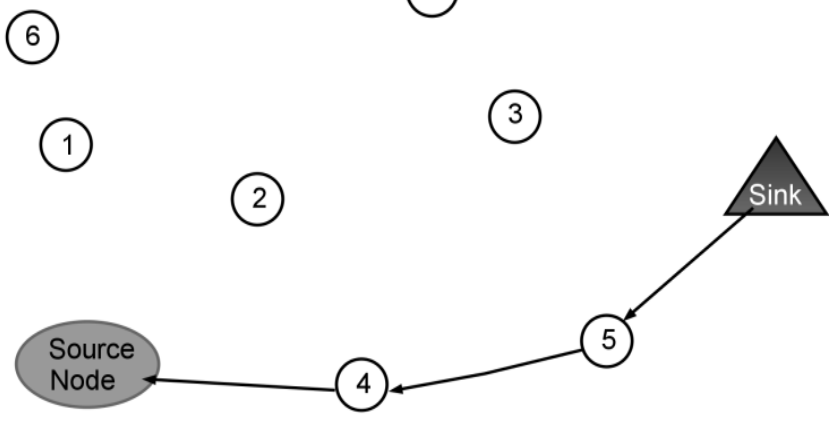

(9)

Figure.3 Warning packets

\section{- Warning Packet:}

The sink combines received parts to find information about detected event. If one or many parts missing, sink sends a warning packets toward the source node $s$ (See Fig. 3) then s sends back the missing parts.

\section{Experimentations and results}

This section evaluates the performance of the proposed routing algorithm based on the TLBO. We adopt the same conditions to have good visualization capabilities for the experimental and comparison purpose.

\subsection{Experimental data}

We perform simulations in MATLAB under 64 bits Windows operating system. Experiments are conducted on a computer with Intel(R) i5 $3: 20 \mathrm{GHz}$ CPU, and 4GB of RAM. The parameter settings used in the simulations are shown in Table 1.

The radio energy dissipation model used is "First Order Radio Model" of Heinzelman et al. [21] mentioned in Fig. 4.
Table 1. Simulations parameters

\begin{tabular}{|l|l|}
\hline Parameter & Value \\
\hline No. of nodes & $10,20,30,40, \ldots, 90,100$ \\
\hline Simulation area & \multicolumn{2}{|c|}{$200 \mathrm{~m}^{2}, 300 \mathrm{~m}^{2}, \ldots 1000 \mathrm{~m}^{2}$} \\
\hline Topology & Flat topology \\
\hline Network type & Static network \\
\hline Deployment type & Random deployment \\
\hline Propagation model & Free space radio propagation model \\
\hline Energy model & First Order Radio Model [21] \\
\hline Initial node energy & 10 Joules \\
\hline No. of simulations & 10 runs \\
\hline
\end{tabular}

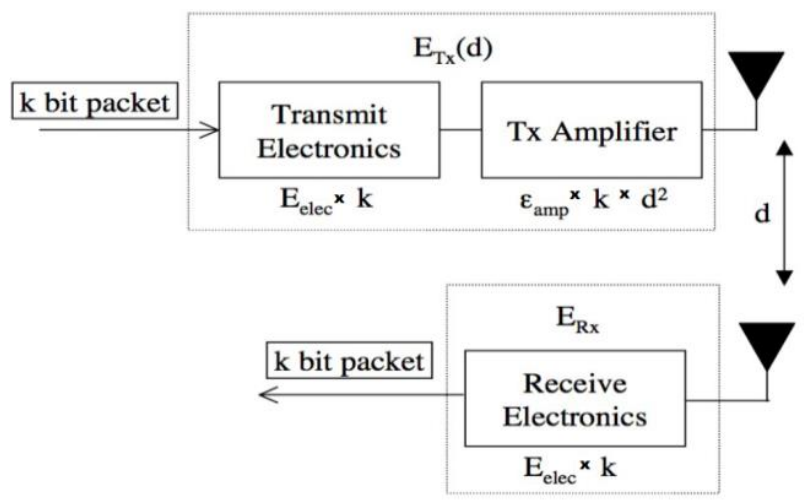

Figure 4: First order radio model [21].

The main parameters of the wireless sensor network model are: the initial energy for each node is $10 \mathrm{~J}$, energy of electronic transmission $E_{\text {elec }}=$ $50 \mathrm{~nJ} / \mathrm{bit}$ and amplification $\varepsilon a m p=100 \mathrm{~nJ} / \mathrm{bit} /$ $m^{2}$.

In order to monitor a static phenomenon, we deploy randomly a number of nodes varied according to the coverage area. A source node detects an event then, using the routing approaches transmits the information to the sink. The sink location is known for the source node (if the sink is not in the coverage area of the source node).

The experiments performed to evaluate the TLBO approach proposed and to illustrate its performance against the ACO approaches [8, 15]. The following section shows the results found.

\subsection{Obtained results and comparison}

In this section, we present simulation results that evaluate the proposed approach based on TLBO and validate its ability of well approximating the energy consumption in the WSN. In addition two wellknown ACO based WSN routing algorithms, were used to make comparisons; EEABR and IACO.

The Fig. 5 shows the results found for the routing approaches ACO, EEABR and TLBO. The average residual energy increases relatively to the variation of number of the packets received. It is 


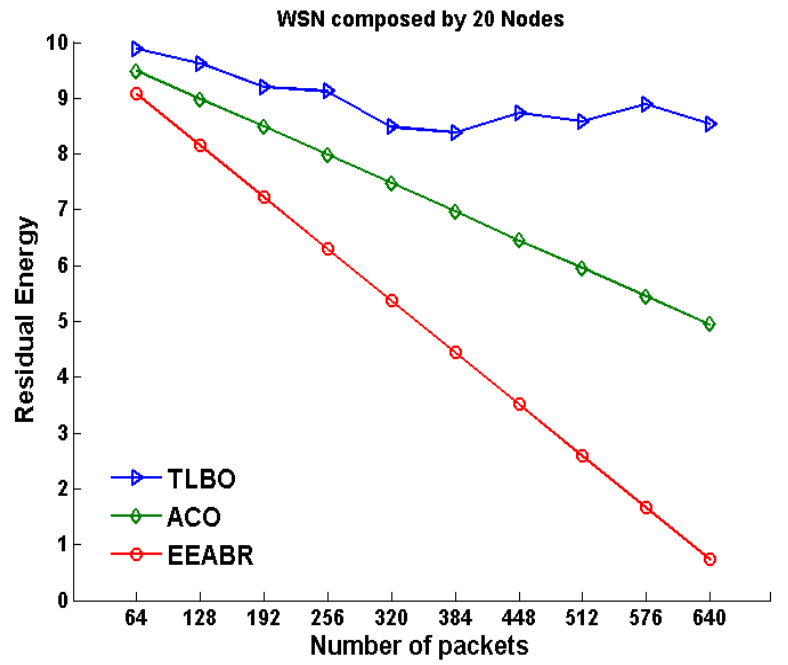

(a)

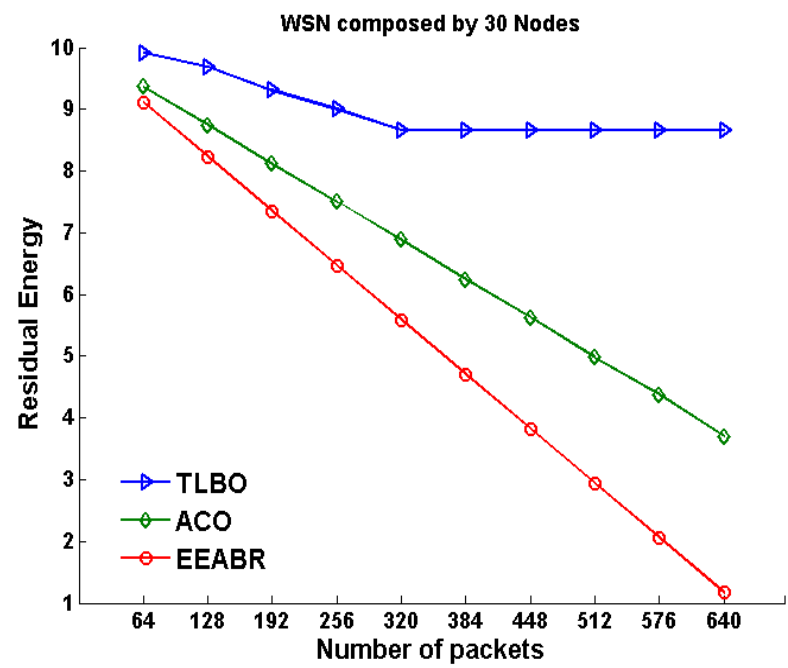

(b)

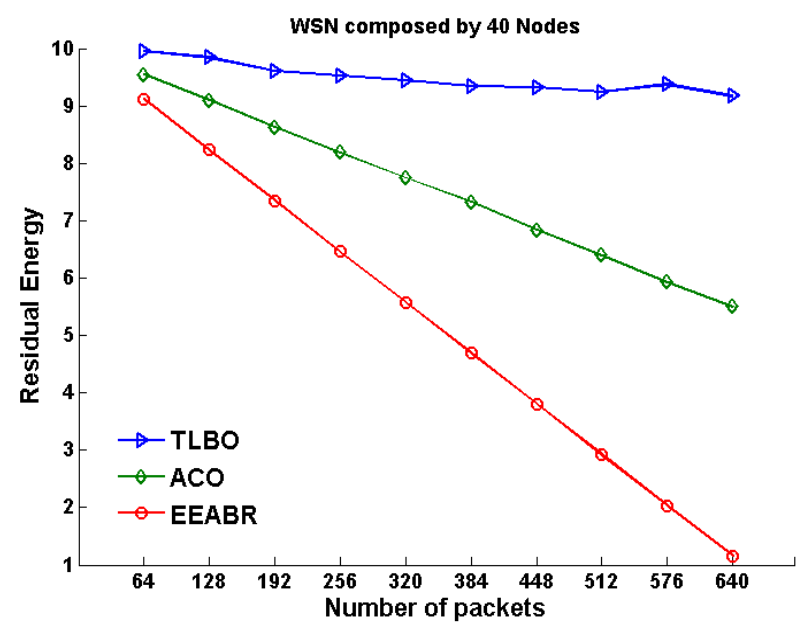

(c)

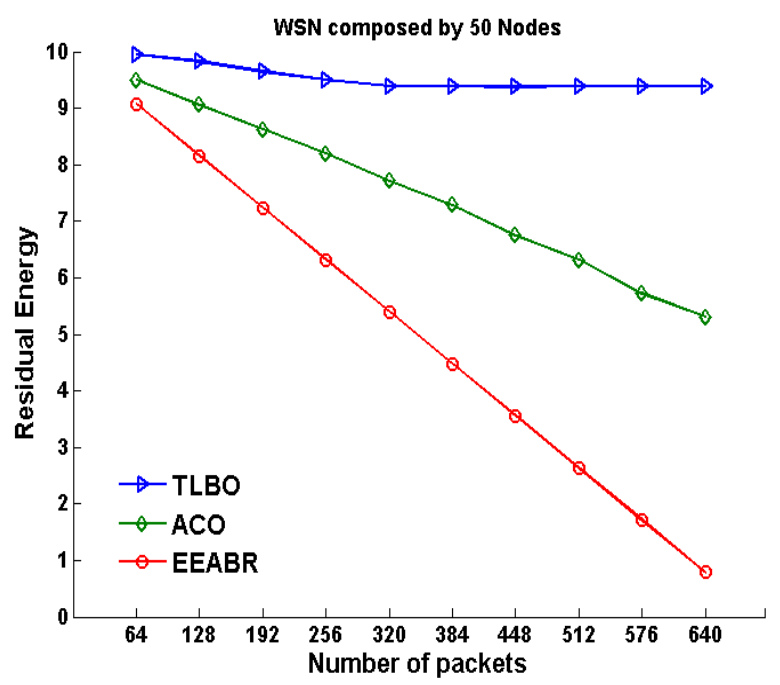

(d)

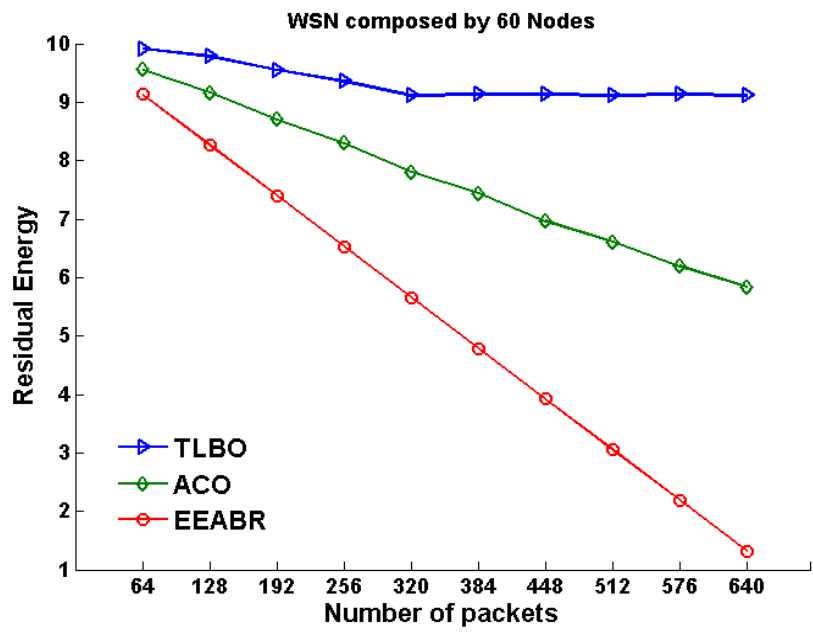

(e)

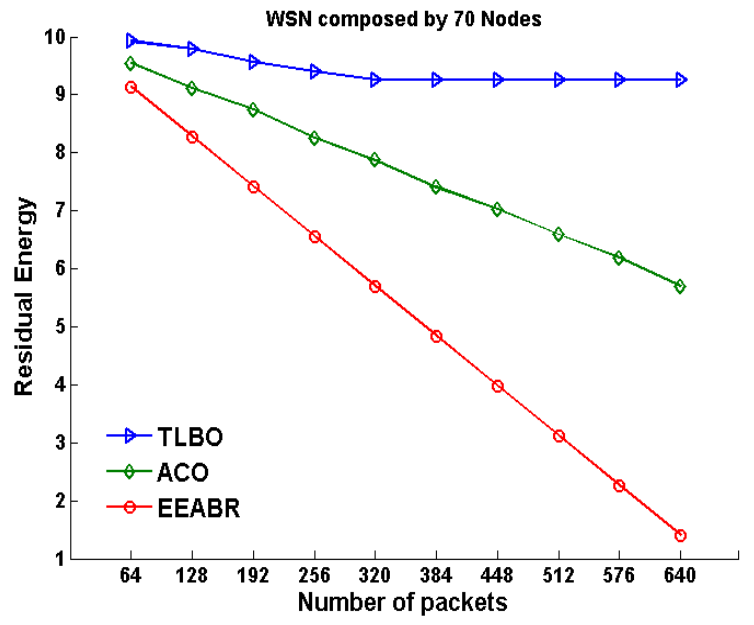

(f) 


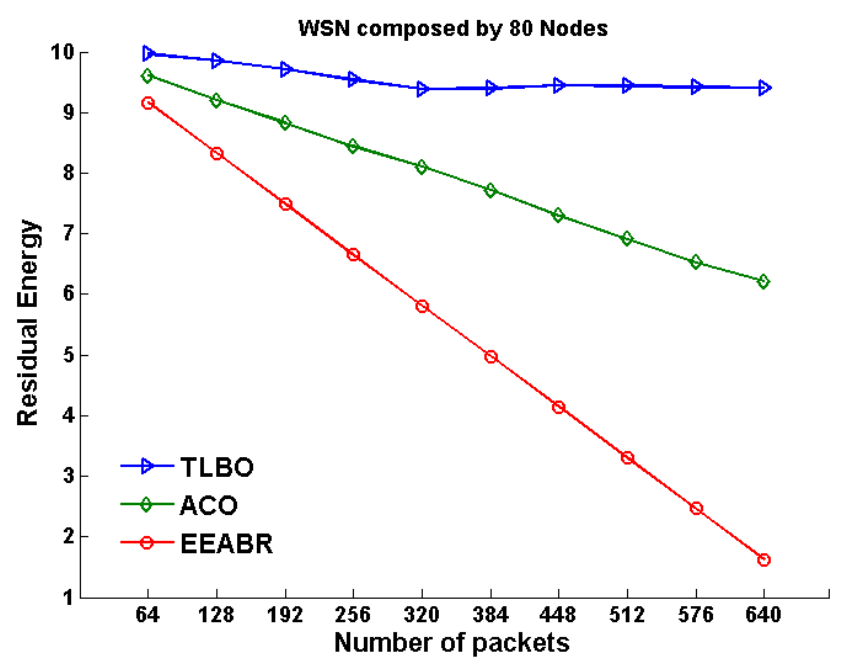

(g)

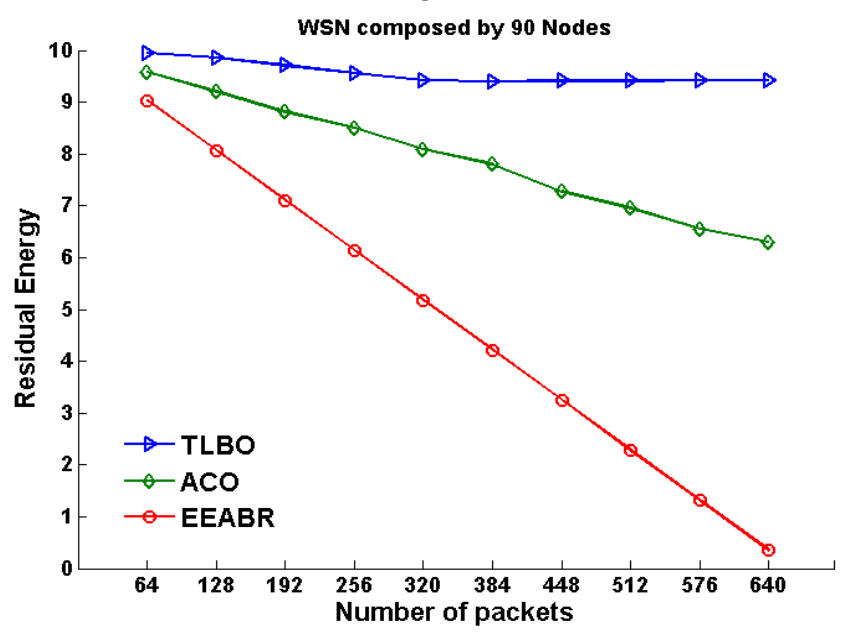

(h)

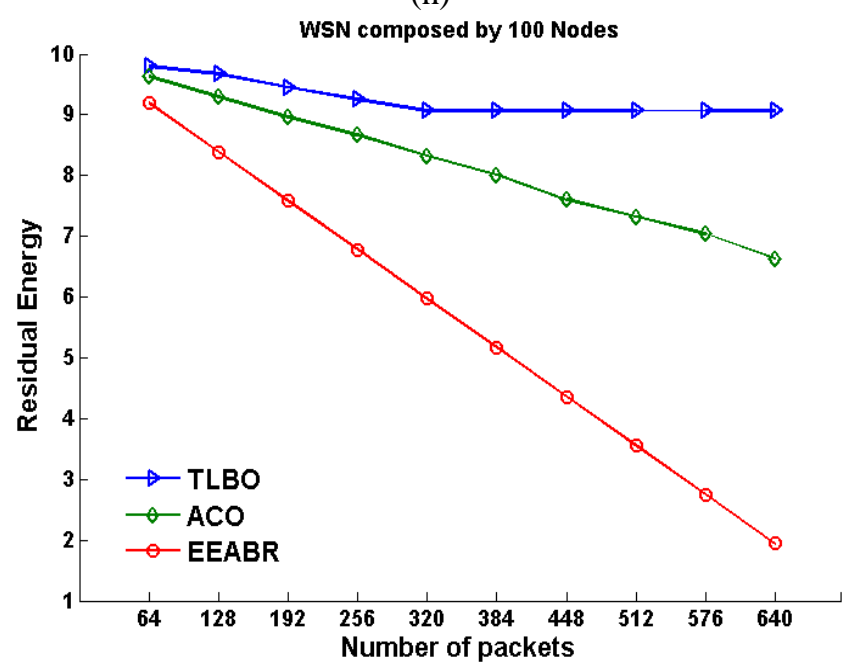

(i)

Figure. 5 Simulation results for different WSNs: (a) 20 nodes, (b) 30 nodes, (c) 40 nodes, (d) 50 nodes, (e) 60 nodes, (f) 70 nodes, (g) 80 nodes, (h) 90 nodes, and (i) 100 nodes

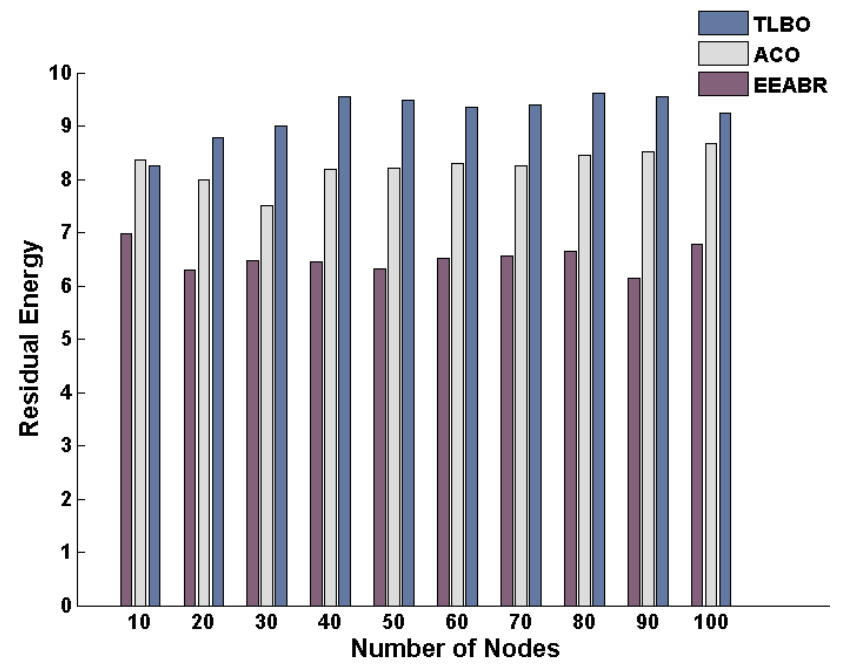

Figure.6 Average residual energy after 256 packets are received

evident to conclude from the same figure that the TLBO approach results exceed greatly the IACO and the EEABR, even when the number of nodes changes. The strategy of TLBO used to choose the optimal path, lets the data reach the sink faster by low energy consumption. Overall, comparing TLBO and ACO metaheuristics for routing, the TLBO optimization promises better results.

Fig. 6 shows the average residual energy after 256 packets received by the sink node for different WSNs having various numbers of nodes. From the Fig .6, it is seen that the difference in energy levels increases as the number of nodes in the network grows.

TLBO algorithm has a basic yet important advantage, which is the absence of parameters. Thus, its simplicity makes the adaptation for NP-hard problems easier than other metaheuristics. Ant colony optimization is one of the metaheuristics used for routing problems in WSN and gave good results. However, the number of parameters in ACO makes TLBO better suited for routing in WSNs as illustrate in the Fig. 6.

\subsection{Discussions}

TLBO is a population-based optimization technique, which implements a group of solutions to seek for the optimum solution. Many optimization methods require algorithm parameters like population size, number of generations, elite size, etc. In addition to the common control parameters, different algorithms require their own algorithm specific control parameters that affect the performance of the algorithm. IACO and EEABR require exponent parameters, pheromone evaporation rate and reward factor. Unlike other 
optimization techniques, TLBO approach proposed does not require any algorithm parameters to be tuned, thus making the implementation of TLBO simpler. TLBO is an algorithm-specific parameterless algorithm that uses the best solution of the iteration to change the current solution in the population thereby increasing the convergence rate. TLBO does not divide the population, it uses two different phases: 'Teacher Phase' and 'Learner Phase' and uses the mean value of the population to update the solution.

TLBO algorithm also has not any special mechanism to handle the constraints. Considering this fact, the proposed TLBO approach for routing in wireless sensor networks is better than the IACO and the EEABR regarding the algorithms implementation and adaptation to the routing problem. In addition as illustrated by the results found in the Fig. 5 and 6, the TLBO approach optimize the energy in different WSNs better than the other approaches.

\section{Conclusion}

This paper presents a new optimization approach based on a metaheuristic inspired by teacher and learners behaviours (TLBO). Teaching learning based optimization is a recent metaheuristic proposed for continuous optimization problems. TLBO is an algorithm-specific parameter-less algorithm considering this fact, TLBO is efficiently adapted to the routing in WSNs; despite it is a discrete optimization problem. The proposed approach provides good results in terms of energy consumption and WSN's lifetime. Various experimentations were performed using the same settings, in order to validate the proposal. We demonstrate that the TLBO approach overcomes widely the ACO based approaches: IACO and EEABR, especially in energy consumption, which is a very crucial factor in the wireless sensors networks.

In the near future, we engage to implement our algorithm in a real wireless sensor network environment in order to further investigate the efficiency of our method.

\section{References}

[1] N. Xu, "A survey of sensor network applications", IEEE Communications Magazine, Vol.40, pp.102-114, 2002.

[2] C. Zhao, C. Wu, X. Wang, B.W.K. Ling, K.L. Teo, J.M. Lee, and K.H. Jung, "Maximizing lifetime of a wireless sensor network via joint optimizing sink placement and sensor-to-sink routing", Applied Mathematical Modelling, Vol. 49, pp. 319-337,2017.

[3] A. Gogu, D. Nace, A. Dilo, and N. Mertnia, "Optimization problems in wireless sensor networks", In: Proc. of International Conf. Complex, Intelligent and Software Intensive Systems (CI-SIS), pp.302-309, 2011.

[4] P.S. Mann and S. Singh, "Artificial bee colony metaheuristic for energy-efficient clustering and routing in wireless sensor networks" Soft Computing, pp.1-14, 2016.

[5] S. Hussain, A.W. Matin, and O. Islam, "Genetic algorithm for energy efficient clusters in wireless sensor networks", In: Proc. of International Conf. Information Technology: New Generations, pp.147-154, 2007.

[6] A. El Ghazi and B. Ahiod, "Particle swarm optimization compared to ant colony optimization for routing in wireless sensor networks", In: Proc. of International Conf. the Mediterranean Conference on Information \& Communication Technologies'15, pp.221-227, 2016.

[7] B. Zeng and Y. Dong, "An improved harmony search based energy-efficient routing algorithm for wireless sensor networks" Applied Soft Computing, pp.135-147, 2016.

[8] A. El Ghazi, B. Ahiod, and A. Ouaarab, "Improved ant colony optimization routing protocol for wireless sensor networks", In: Proc. of International Conf. Networked Systems, pp.246-256, 2014.

[9] S. Okdem and D. Karaboga, "Routing in wireless sensor networks using an ant colony optimization (aco) router chip", Sensors, Vol.9, No.2, pp.909921, 2009.

[10] K. Fathima and K, Sindhanaiselvan, "Ant colony optimization based routing in wireless sensor networks", International Journal of Advanced Networking and Applications, Vol.4, No.4, pp. 1686, 2013.

[11] Y. Zhang, L.D. Kuhn, and M.P. Fromherz, "Improvements on ant routing for sensor networks", In: Proc. of International Conf. Ant Colony Optimization and Swarm Intelligence, Vol.3172, pp.154-165, 2004.

[12] Y. Lu, G. Zhao, and F. Su, "Adaptive ant-based dynamic routing algorithm", In: Proc. of International Conf. World Congress on Intelligent Control and Automation (WCICA), Vol.3, pp. 2694-2697, 2004.

[13] R. Ghasem Aghaei, M.A. Rahman, W. Gueaieb, and A. El Saddik, "Ant colony-based reinforcement learning algorithm for routing in wireless sensor networks", In: Proc. of International Conf. Instrumentation and 
Measurement Technology Conference Proceedings (IMTC), pp.1-6, 2007.

[14] Y.f. Wen, Y.q. Chen, and M. Pan, "Adaptive ant-based routing in wireless sensor networks using energy* delay metrics", Journal of Zhejiang University-SCIENCE A, Vol.9, No., pp.531-538, 2008.

[15] T. Camilo, C. Carreto, J. Silva, and F. Boavida, "An energy-efficient ant-based routing algorithm for wireless sensor networks", Ant Colony Optimization and Swarm Intelligence, pp. 49-59, 2006.

[16] R. Rao, V. Savsani, and D. Vakharia, "Teachinglearning-based optimization: A novel method for constrained mechanical design optimization problems", Computer-Aided Design, Vol.43, No.3, pp.303-315, 2011.

[17] R. Rao, V. Savsani, and D. Vakharia, "Teachinglearning-based optimization: an optimization method for continuous non-linear large scale problems", Information Sciences, Vol.183, No.1, pp.1-15, 2012.

[18] M. Dorigo and G. Di Caro, "Ant colony optimization: a new metaheuristic", In: Proc. of International Conf. Congress on Evolutionary Computation (CEC), Vol.2, pp.1-8, 1999.

[19] V. Toğan and A. Mortazavi, "Sizing optimization of skeletal structures using teaching-learning based optimization" $A n$ International Journal of Optimization and Control: Theories \& Applications (IJOCTA), Vol.7, No.2, pp.130-141, 2017.

[20] B.D Raja, R. Jhala, and V. Patel, "Multiobjective optimization of a rotary regenerator using tutorial training and self-learning inspired teaching-learning based optimization algorithm (ts-tlbo)", Applied Thermal Engineering, Vol. 93, pp. 456-467, 2016.

[21] W.R. Heinzelman, A. Chandrakasan, and H. Balakrishnan, "Energy-efficient communication protocol for wireless microsensor networks", In: Proc. of International Conf. 33rd Annual Hawaii International Conference on System Sciences, pp.1-10, 2000. 